\title{
Holographic approach to the light-by-light contribution to the muon (g-2)
}

\author{
Luigi Cappiello $0^{1,2, a}$ \\ ${ }^{1}$ Dipartimento di Fisica "Ettore Pancini", Universitá di Napoli "Federico II" \\ ${ }^{2}$ INFN-Sezione di Napoli, Italy
}

\begin{abstract}
We discuss how holographic models of QCD are used to study the the hadronic light-by-light (HLbL) scattering amplitude contribution to the muon anomalous magnetic moment. After a brief description of the various models, we focus on a particular one, introduced by Hirn and Sanz, emphasizing the role it assigns to the pion, and the vector and-axial vector resonances. We review how the parameters of the model are fixed by imposing condition on the low energy and the deep Euclidean limit of two-point correlators of QCD vector and axial vector currents. We then focus on the evaluation of the three-point axial-vector-vector current correlator and the pion anomalous transition form factor to be used in the one-pion exchange HLbL diagram and study its asymptotic properties. Finally, we present preliminary results on the four vector current correlator that defines the Hadronic Light-by-Light tensor. We find that axial-vector resonances play an important rule in recovering the correct asymptotic behaviour predicted by QCD at large Euclidean momenta.
\end{abstract}

\section{Introduction}

The present experimental value for the anomalous magnetic moment of the muon, $a_{\mu}=(g-2)_{\mu} / 2$, is given by $a_{\mu}^{\mathrm{EXP}}=11659209.1(5.4)(3.3) \times 10^{-10}[1]$, where statistical errors are the largest source of uncertainties. The experimental error is expected to be reduced by a factor four in next experiments at FNAL [2] and J-PARC [3]. There is a discrepancy of about $3 \sigma$ with the experimental value, which, if confirmed with the precision of $1.6 \times 10^{-10}$ of the foreseen experiments, would rise up to a $6 \sigma$ effect. This has also led to suggest explanations in term of new physics beyond the Standard Model.

Theoretical estimates of $a_{\mu}$ include contributions from many different sectors [4], and among them, the most difficult to evaluate and having so far produced a lot of relevant theoretical work, are those due to non perturbative hadronic physics. This is particularly true for the hadronic contribution to the light-by-light (HLbL) scattering Feynman diagram of Fig. 1, containing the HLbL tensor, defined as the Green's function of four electromagnetic currents evaluated in QCD :

$$
\begin{aligned}
& \Pi^{\mu \nu \lambda \sigma}\left(q_{1}, q_{2}, q_{3}\right)=-i \int d^{4} x d^{4} y d^{4} z e^{-i\left(q_{1} \cdot x+q_{2} \cdot y+q_{3} \cdot z\right)} \\
& \quad \times<0\left|T\left\{j_{\mathrm{em}}^{\mu}(x) j_{\mathrm{em}}^{\nu}(y) j_{\mathrm{em}}^{\lambda}(z) j_{\mathrm{em}}^{\sigma}(0)\right\}\right| 0>
\end{aligned}
$$

General arguments, based on chiral symmetry and the Large- $N_{c}$ limit of QCD, have identified the one-pion exchange as the dominant contribution. The corresponding Feynman diagrams are shown in Fig. 2, and contain a neutral pion transition form factor (TFF) at each pion-photonphoton interaction vertex.

\footnotetext{
ae-mail: luigi.cappiello(at)na.infn.it
}

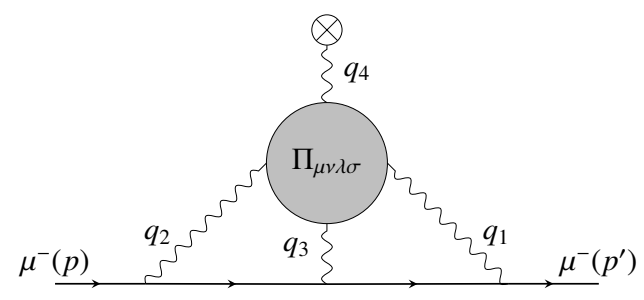

Figure 1. The HLbL diagram. The blob represent the HLbL tensor.

Many theoretical efforts have been devoted to the evaluation of the hadronic HLbL conttribution to $a_{\mu}$, using, for instance, the Extended Nambu-Jona Lasinio model [5, 6], or modeling the TFF using (extended versions of the) Vector Meson Dominance (VMD) [7-11]. In fact, the simplest possibility of using of a constant TFF, corresponding to the constant WZW anomalous vertex, would give a divergent contribution to $a_{\mu}$, requiring a cut-off on momentum integral. An important result of Chiral Symmetry and Large$N_{c}$, is the universality of the coefficient of the leading $\log ^{2}$ divergence [12].
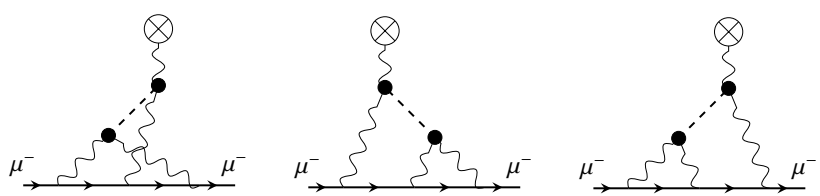

Figure 2. The three one-pion exchange HLbL diagrams. The dashed line denotes the pion propagator and the black dots the TFF at each anomalous $\pi^{0} \gamma^{*} \gamma^{*}$ vertex. 
VMD, and its developments, such as lowest meson dominance (LMD) [13] and the LMD+V model [14] have been very successful in hadron physics in two respect: first, giving excellent predictions for low energy hadronic physics, extending the results obtained for pions with Chiral Lagrangians, secondly, improving the matching with the high energy behaviour. Indeed, the study of UV constraints from perturbative QCD on the hadronic HLbL contribution has become more and more important.

A new UV constraint on the e.m. four-point function in (1), based on a QCD short-distance OPE relation and holding in the particular kinematic regime, where two of the (Euclidean) photon momenta are much bigger than the third (the fourth photon is taken to be the soft external one), was found by Melnikov and Vainshtein (MV) in Ref.[15]. A much milder decrease at large momenta of the e.m. four-point function, is predicted by the MV constraint and this fact has brought tension on models based on the pion-pole exchange. The same authors of Ref.[15], in order to comply with the asymptotic behaviour, were led to propose a pion-pole model, with a (pointlike) constant WZW form factor at the soft pion vertex, while a full TFF is attached to vertex with the two off-shell s. A a rather asymmetric choice which fails to recover crossing symmetries. An alternative approach, proposed in [4], led to fullfill the MV constraint with a more complicated LMV+V form of TFF at both vertices, but at the cost of adding more parameters into the game, whose physical significance is more contrived.

The MV constraint has consequences for the evaluation of the contribution of one-particle axial-vector resonance exchange, with, for instance, similar asymmetric proposals for the use of the corresponding form factor [15]. Nevertheless, the contribution to $a_{\mu}$ has been estimated to be much smaller than that due to the pion-pole exchange.

All these relevant issues have led to an increase of of the estimated value of the HLbL scattering contribution to $a_{\mu}$ finally leading to the two reference numbers reported in the literature, $a_{\mu}^{\mathrm{HIBL}}=(11.6 \pm 4.0) \times 10^{-10}[4]$ and $(10.5 \pm$ 2.5) $\times 10^{-10}[16]$.

We shall first give a brief summary of HQCD models, (see the seminal papers [17-21]) which are based on classical Lagrangians, although defined on a (curved) fivedimensional (5D) space, and are constructed so to approximate well the almost conformal behavior of QCD at large momenta. Non-perturbative features of Large- $N_{c}$ QCD, such as, for instance, the appearance of an infinite number of narrow resonance, saturating amplitude channels, can be naturally encoded in HQCD models, which then provide explicit analytic expressions for correlation functions of QCD operators, covering the full energy range. Moreover, although depending on few independent parameters, they produce surprisingly good results at low energy, with predictions comparable with those of Chiral Lagrangians, and vector meson dominance.

It is clear, however, that HQCD models are not aimed at producing accurate physics predictions, but rather to get hints in the study of QCD phenomena, which could be suggestive for more sound attempts, e.g. lattice calculations.

HQCD models inspired by the gauge/gravity duality have been already considered for the evaluation of HIBL [22, 23]. However, in those papers HQCD models were used, as it was the case for many other hadronic models, to obtain (analytic) expressions for the TFF, which were then used to evaluate the pion-pole contribution to $a_{\mu}$, shown in Fig.1. (QCD four-point amplitudes, related to the $\Delta I=$ $1 / 2$ rule, and the $B_{K}$ mixing parameter, obtained from the same HQCD model we consider here, have been studied in $[24,25])$.

We shall present also some preliminary results about using one of these HQCD model to get informations directly on the HLbL tensor. In particular, as HQCD models approximate well the pQCD limit, we shall focus on the prediction on the asymptotic behavior of the HlbL tensor in the MV limit. The details will be presented in a forthcoming paper[26].

The plan of the paper is as follows. In Sect.2, after a general introduction to HQCD models, we focus on the HQCD model proposed in Ref.[20] and the role the model assigns to the pion and the vector and-axial vector resonances. In Sect.3, we review how the parameters of the model are fixed, imposing matching condition on the asymptotic limit of two-point correlators of QCD vector and axial currents currents. In Sect.4, we shall focus on the evaluation of AVV three-point axial-vector-vector current correlator, in order to extract the pion TFF to be used in the one-pion exchange HLbL diagram and we study its asymptotic properties. Finally, in Sect.5 we give some preliminary results on the prediction of the HQCD model for the four vector current correlator that defines the HLbL tensor, and its asymptotic limits.

\section{Holographic models of QCD}

HQCD models have been constructed along the lines of the original conjectured AdS/CFT duality (equivalence) between a four dimensional (4D) (conformal) Large- $\mathrm{N}_{c}$ gauge theory at strong coupling and a (classical) fivedimensional (5D) field theory in a curved gravitational background with Anti-de-Sitter metric [27], which can be summarized as follows [28, 29]: for every quantum operator $O_{\Delta}(x)$, with scaling dimension $\Delta$, of the $4 \mathrm{D}$ (strong coupled) gauge theory, there exists a corresponding $5 \mathrm{D}$ field $\phi(x, z)$, whose value on the ultraviolet brane (taken at $z=0) \phi(x, 0) \equiv \mathbf{s}(x)$, is identified with the fourdimensional source of $O(x)$. The generating functional of the $4 \mathrm{D}$ theory can be computed from the 5D action evaluated on-shell, i.e.:

$$
\begin{aligned}
\exp (i W[\mathbf{s}(x)]) \equiv\langle 0| & \exp \left(i \int d^{4} x \mathbf{s}(x) O_{\Delta}(x)\right)|0\rangle_{Q C D} \\
& =\exp \left(i S_{5}\left(\phi_{0}(z, x)\right)\right)
\end{aligned}
$$

By varying the action with respect to $\phi_{0}(x)$, one can generate connected $n$-point Green's functions of large- $N_{c}$, 
Table 1. The holographic dictionary

\begin{tabular}{|c|c|}
\hline 4D & $5 \mathrm{D}$ \\
\hline operator $O_{\Delta}(x)$ & dual field $\Phi(x, z)$ \\
\hline source coupled to $O_{\Delta}(x) \mathbf{s}(\mathbf{x}) \leftarrow$ & on-shell $\Phi_{0}(x, z)$ \\
\hline conformal dimension $\Delta$ & mass: $m_{\Phi}^{2}=$ \\
\hline & $(\Delta-p)(\Delta+p-4)$ \\
\hline $\begin{array}{l}U\left(N_{f}\right)_{L} \times U\left(N_{f}\right)_{R} \\
\text { global symmetry }\end{array}$ & $\begin{array}{l}U\left(N_{f}\right)_{L} \times U\left(N_{f}\right)_{R} \\
\text { gauge symmetry }\end{array}$ \\
\hline vector current $\bar{q} \gamma^{\mu} t^{a} q \quad \mathbf{v}_{u}^{a}(x) \leftarrow$ & gauge field $V_{u}^{a}(x, z)$ \\
\hline axial current $\bar{q} \gamma^{\mu} \gamma_{5} t^{a} q \mathbf{a}_{\mu}^{a}(x) \leftarrow$ & gauge field $A_{\mu}^{a}(x, z)$ \\
\hline quark bilinear $\bar{q} t^{a} q \quad \mathbf{s}(x) \leftarrow$ & scalar field $X^{a}(x, z)$ \\
\hline
\end{tabular}

strong-coupled 4D gauge theory.

$$
\left\langle 0\left|T\left\{O_{\Delta}\left(x_{1}\right) O_{\Delta}\left(x_{2}\right) \cdots O_{\Delta}\left(x_{n}\right)\right\}\right| 0\right\rangle \sim \frac{\delta^{n} S_{5}\left(\phi_{0}\right)}{\delta \mathbf{s}\left(x_{1}\right) \delta \mathbf{s}\left(x_{2}\right) \cdots \delta \mathbf{s}_{0}\left(x_{n}\right)}
$$

In HQCD models, the 5D action describes an Yang-Mills theory with Chern-Simons term in a curved $5 \mathrm{D} \mathrm{AdS}_{5}$ space. The field content dictated by the AdS/CFT correspondence is summarized in Table 1. HQCD models differ in the way fundamental low energy properties such as confinement and chiral symmetry breaking $(\chi \mathrm{SB})$ of QCD are realzed. In Hard-Wall (HW) models, confinement corresponds to a sharp cut-off of the extra dimension to a finite size of about $\Lambda_{Q C D}^{-1}$. In Soft-Wall models, confinament is driven by a non trivial dilaton profile. It should be mentioned that, while most of the HQCD models has been engineered using a phenomenological bottom-up approach, there is at least one top-bottom model, the Sakai-Sugimoto model (SS), that has been derived as the low energy limit of a string (D-brane) setting.

The 5D action of HQCD models is generically given by:

$$
S_{5}=S_{\mathrm{YM}}+S_{\mathrm{X}}+S_{\mathrm{CS}}
$$

with

$$
\begin{gathered}
S_{\mathrm{YM}}=-\int d^{4} x \int_{0}^{z_{0}} d z e^{-\Phi(z)} \frac{1}{8 g_{5}^{2}} w(z) \operatorname{tr}\left[\mathcal{F}_{L}^{2}+\mathcal{F}_{R}^{2}\right] \\
S_{X}=\int d^{4} x \int_{0}^{z_{0}} d z e^{-\Phi(z)} w(z)^{3} \operatorname{tr}\left[D^{M} X D_{M} X^{\dagger}+V\left(X^{\dagger} X\right)\right] \\
S_{\mathrm{CS}}=\frac{N_{c}}{24 \pi^{2}} \int \operatorname{tr}\left(\mathcal{A}_{L} \mathcal{F}_{L}^{2}-\frac{i}{2} \mathcal{A}_{L}^{3} \mathcal{F}_{L}-\frac{1}{10} \mathcal{A}_{L}^{5}\right)-(L \rightarrow R) .
\end{gathered}
$$

In these equations, the $5 \mathrm{D}$ metric is $d s_{5}^{2}=$ $w(z)^{2}\left(d x_{\mu}^{2}-d z^{2}\right)$, with $w(z)=1 / z$, for $A d S$. $\mathcal{F}_{M N}=\partial_{M} \mathcal{A}_{N}-\partial_{N} \mathcal{A}_{M}-i\left[\mathcal{A}_{M}, \mathcal{A}_{N}\right]$ and $\mathcal{A}_{L, R}=V \mp A$. In HW models, $z$ has a sharp cut-off at a finite value of $z_{0}$, while in SW models, there is a background dilaton field $\Phi(z)=-\kappa^{2} z^{2}$. In any model this produce the appearance of infinite towers vector and axial-vector Kaluza-Klein modes, to be identified with the massive (stable) resonances predicted in the Large- $N_{c}$ limit of QCD.

HW models differ in the way $\chi \mathrm{SB}$ is implemented. In the HW1 models, the 5D scalar field $X(x, z)$, dual to $\bar{q} q$, induces $\chi \mathrm{SB}$, by acquiring a non trivial 5D profile $X=v(z)$, while in HW2, $\chi \mathrm{SB}$ broken by different boundary conditions for $V_{\mu}$ and $A_{\mu}$ on the IR wall $z_{0}$ and the 4D chiral field $U(x)$ appears as the remnant of non trivial 5D Wilson line of the fifth component of the axial-vector gauge field $A_{z}$.

In the following, we shall focus on the HW2 model proposed by Hirn and Sanz (HS), [20], and analyze twothree- and four point functions which are relevant for the evaluation of the hadronic Light-by-Light contribution to $(g-2)_{\mu}$. The HS model has the advantage of dealing with a rather simple 5D action: there are no scalars in the bulk, so that there is no term $S_{X}$ in the 5D action, no dilaton profile, and hence the $5 \mathrm{D}$ equation of motion is the same for vector and axial-vector gauge fields, depending only on the simple AdS metric $w(z)=1 / z$; solutions are readily expressed in terms of Bessel functions. Different expressions for vector and axial-vector gauge field solutions of the 5D EOM are due to the different $\chi \mathrm{SB}$ boundary conditions imposed at $z_{0}=0$. Moreover, the chiral field $U(x)=\exp \left(2 i \pi(x) / f_{\pi}\right)$ appears in the 4D effective action for pions (or more generically for the Goldstone bosons of QCD spontaneous $\chi \mathrm{SB})$, with $U(x)$ appearing as a non trivial zero mode of the axial gauge-field. Then, the the CS term $S_{C S}$ reproduces the gauged Wess-Zumino-Witten action. Finally, notice that the model has only two independent parameters: the 5D gauge coupling $g_{5}$ and the size of the extradimension $z_{0}$.

\section{Two-point functions: fixing the parameters of HS model}

According to the general recipe (1), two point functions of vector or axial-vector quark currents of QCD are obtained by the following fuctional derivatives of the 5D (on-shell) action expressed in terms of the boundary values $\mathbf{v}_{\mu}(x)$ and $\mathbf{a}_{\mu}(x)$ of the solution of the 5D EOM of vector an axialvector gauge fields

$$
\begin{aligned}
& \left\langle 0\left|T\left\{J_{V}^{\mu}(x) J_{V}^{v}(y)\right\}\right| 0\right\rangle \sim \frac{\delta^{2} S_{5}}{\delta \mathbf{v}_{\mu}(x) \delta \mathbf{v}_{v}(y)} \\
& \left\langle 0\left|T\left\{J_{A}^{\mu}(x) J_{A}^{v}(y)\right\}\right| 0\right\rangle \sim \frac{\delta^{2} S_{5}}{\delta \mathbf{a}_{\mu}(x) \delta \mathbf{a}_{v}(y)}
\end{aligned}
$$

The result for the scalar functions $\Pi_{V, A}$ defined by

$$
\begin{aligned}
& 2 i \int d^{4} x e^{i q \cdot x}\left\langle T\left\{J_{V, A}^{a, \mu}(x) J_{V, A}^{b, v}(0)\right\}\right\rangle \\
& =\delta^{a b}\left(q^{\mu} q^{v}-q^{2} g^{\mu v}\right) \Pi_{V, A}\left(q^{2}\right)
\end{aligned}
$$

can be represented graphically as follows

$$
\begin{aligned}
& \Pi_{V}\left(q^{2}\right)=L_{10}^{L_{10} H_{1}}+\frac{q^{2}}{g_{5}^{2}} \int_{0}^{z 0} d z \int_{0}^{z 0} d z^{\prime} \underset{\rho, \rho^{\prime}, \ldots}{\otimes} \otimes \\
& =\left.\frac{1}{q^{2} g_{5}^{2}} \partial_{z} \partial_{z} \otimes \frac{V}{\otimes} \otimes\right|_{z=z^{\prime}=0}
\end{aligned}
$$


and

$$
\begin{gathered}
\Pi_{A}\left(q^{2}\right)=f_{\pi}^{2}+f_{\pi}^{f_{\pi}}+L_{i 10}^{L_{10}, H_{1}} \\
+\frac{q^{2}}{g_{5}^{2}} \int_{0}^{z 0} d z \int_{0}^{z 0} d z^{\prime} \underbrace{A}_{a_{1}, a_{1}^{\prime}, \ldots} \otimes \\
=\left.\frac{1}{q^{2} g_{5}^{2}} \partial_{z} \partial_{z^{\prime}} \otimes \frac{A}{=} \otimes\right|_{z=z^{\prime}=0}
\end{gathered}
$$

While the first lines in the expressions for $\Pi_{V}\left(q^{2}\right)$ and $\Pi_{A}\left(q^{2}\right)$ above show the infinite sums on resonances, with the pion appearing as the massless pole in $\Pi_{A}\left(q^{2}\right)$, the last lines indicate that $\Pi_{V}\left(q^{2}\right)$ and $\Pi_{A}\left(q^{2}\right)$ can be written in terms of the respective 5D Green functions, $G_{V, A}\left(z, z^{\prime}, q^{2}\right)$, showing that the HS model gives analytical expressions for the two-point function on the full momentum range.

In particular, comparing the expansions for deep Euclidean momentum $Q^{2}=-q^{2}$ with the parton loop of $\mathrm{pQCD}$, one has

$\Pi_{V, A}\left(-Q^{2}\right)=-\frac{1}{g_{5}^{2}}\left(\log \frac{Q^{2}}{\mu^{2}}\right)+\cdots=-\frac{N_{c}}{12 \pi^{2}}\left(\log \frac{Q^{2}}{\mu^{2}}\right)+O\left(\frac{1}{Q^{4}}\right)$

which fixes

$$
g_{5}^{2}=\frac{12 \pi^{2}}{N_{c}}
$$

The remaining parameter, $z_{0}$, is usually fixed by imposing that the mass of the first vector resonance coincide with the mass of $776 \mathrm{MeV}$ of the $\rho$ meson:

$$
m_{\rho}=\frac{\gamma_{0,1}}{z_{0}}
$$

where $\gamma_{0,1} \simeq 2.4$ is the first zero of the Bessel function $J_{0}(x)$. This gives $z_{0}=3 \cdot 1 \mathrm{GeV}^{-1}$.

Any other physical quantity is predicted once we fix $N_{c}=3$. In particular, we mention the value of the first axial-vector mass $m_{a_{1}}=1263 \mathrm{MeV}$, very close to the experimental value, while the pion decay constant, which is in the HW2 model is given by

$$
f_{\pi}^{2}=\frac{N_{c}}{6 \pi z_{0}^{2}}
$$

turns out to underestimate the physical value.

\section{Three-point function: the pion anomalous transition form factor}

A fundamental quantity in the evaluation of the leading HLbL to the muon anomalous magnetic moment, is the pion anomalous transition form factor (TFF),

$$
\begin{aligned}
& \int d^{4} x e^{-i q_{1} \cdot x}\left\langle\pi^{0}\left(q_{1}+q_{2}\right)\left|T\left\{J_{e . m .}^{\mu}(x) J_{e . m .}^{v}(0)\right\}\right| 0\right\rangle \\
& =\epsilon^{\mu \nu \rho \sigma} q_{1 \rho} q_{2 \sigma} \mathcal{F}_{\pi^{0} \gamma^{*} \gamma^{*}}\left(q_{1}^{2}, q_{2}^{2}\right) .
\end{aligned}
$$

For real photons one has the constraint

$$
\mathcal{F}_{\pi^{0} \gamma^{*} \gamma^{*}}(0,0)=\frac{N_{c}}{12 \pi^{2} f_{\pi}} \text { (pointlike WZW vertex) }
$$

Defining the normalized TFF

$$
K\left(Q_{1}^{2}, Q_{2}^{2}\right) \equiv \mathcal{F}_{P \gamma^{*} \gamma^{*}}\left(Q_{1}^{2}, Q_{2}^{2}\right) / \mathcal{F}_{P \gamma^{*} \gamma^{*}}(0,0),
$$

with Euclidean momenta, $Q_{1,2}^{2}=-q_{1,2}^{2}$, such that $K(0,0)=$ 1 , one has, in the HS model that

$$
K\left(Q_{1}^{2}, Q_{2}^{2}\right)=-\int_{0}^{z_{0}} v\left(z, Q_{1}^{2}\right) v\left(z, Q_{2}^{2}\right) \partial_{z} \alpha(z) d z
$$

where $\alpha(z)=1-z / z_{0}$, and $v\left(z, q^{2}\right)=$ $\left.\left(-1 / z^{\prime}\right) \partial_{z^{\prime}} G_{V}\left(z, z^{\prime} ; Q^{2}\right)\right|_{z^{\prime} \rightarrow 0}$ is the so called vector bulk-to-boundary propagator.

At low momenta, the slope $\widehat{\alpha}$ defined in

$$
\begin{aligned}
& K\left(Q_{1}^{2}, Q_{2}^{2}\right)=1+\widehat{\alpha}\left(Q_{1}^{2}+Q_{2}^{2}\right) \\
& +\widehat{\beta} Q_{1}^{2} Q_{2}^{2}+\widehat{\gamma}\left(Q_{1}^{4}+Q_{2}^{4}\right)+\ldots
\end{aligned}
$$

its value $\widehat{\alpha}=-1.80 \mathrm{GeV}^{-2}$ in good agreement with the world average $-1.84(17) \mathrm{GeV}^{-2}$ [30]. For single and double photon virtualities, at large Euclidean $Q^{2}$ momenta (with $N_{c}=3$ ):

$$
K^{p Q C D}\left(Q^{2}, 0\right) \simeq \frac{8 \pi^{2} f_{\pi}^{2}}{Q^{2}} \quad K^{p Q C D}\left(Q^{2}, Q^{2}\right) \simeq \frac{8 \pi^{2} f_{\pi}^{2}}{3 Q^{2}}
$$

and coincide with the predictions of pQCD.

The TFF enters in the HLbL one pion exchange Feynman diagrams of Fig.2, shown in the Introduction, and fixing the parameters of the model so to obtain the physical values of $\mathcal{F}_{\pi^{0} \gamma^{*} \gamma^{*}}(0,0)$ and of the mass of the $\rho$ meson, one gets the result

$$
a_{\mu}^{H L b L, \pi^{0}}=5.7 \times 10^{-10}
$$

which is comparable with the results shown in Table 2.

Table 2. Values of the one-pion exchange obtained using different pion TFF

\begin{tabular}{c|c|c}
\multicolumn{3}{c}{$a_{\mu}^{\mathrm{HLbL}, \pi^{0}} \times 10^{-10}$} \\
\hline \hline VMD & 5.7 & {$[11]$} \\
\hline LMD+V & 6.3 & {$[11]$} \\
\hline \hline DIP & 6.58 & {$[23]$} \\
\hline$\langle$ HQCD's $\rangle$ & $5.9(2)$ & {$[31]$} \\
\hline \hline DVR interp. & $5.64(25)$ & {$[32]$} \\
\hline Lattice & $5.97 \pm 0.23$ & {$[33]$} \\
\hline
\end{tabular}

In Table 2, the first two entries refer to the use of phenomenological TFF values (VMD, LMD+V) based on generalized vector meson dominance [11], the third value was obtained by using a HQCD approach with different interpolator, satisfying additional high energy constraints [23], the fourth value is the average of values of $a_{\mu}$ on the whole set of HQCD models we mentioned in the Section 2 as done in the more recent analysis [31], the fifth reference uses another phenomenological inspired interpolator [32] and finally the last raw refers to a recent lattice study [33]. 


\section{Four-point function: the hadronic light-by-light tensor}

The fundamental object entering the HLbL contribution to $a_{\mu}$ is the HLbL tensor defined in terms of the correlator of four elecromagnetic quark currents, defined in eq.(1). Of the 138 independent Lorentz structures in which it can decomposed

$$
\begin{aligned}
& \Pi^{\mu \nu \lambda \sigma}=g^{\mu v} g^{\lambda \sigma} \Pi^{1}+g^{\mu \lambda} g^{v \sigma} \Pi^{2}+g^{\mu \sigma} g^{v \lambda} \Pi^{3} \\
+ & \sum_{i, j=1,2,3}\left(g^{\mu v} q_{i}^{\lambda} q_{j}^{\sigma} \Pi_{i j}^{4}+g^{\mu \lambda} q_{i}^{v} q_{j}^{\sigma} \Pi_{i j}^{5}+g^{\mu \sigma} q_{i}^{v} q_{j}^{\lambda} \Pi_{i j}^{6}\right. \\
+ & \left.g^{v \lambda} q_{i}^{\mu} q_{j}^{\sigma} \Pi_{i j}^{7}+g^{v \sigma} q_{i}^{\mu} q_{j}^{\lambda} \Pi_{i j}^{8}+g^{\lambda \sigma} q_{i}^{\mu} q_{j}^{v} \Pi_{i j}^{9}\right) \\
+ & \sum_{i, j, k, l=1,2,3} q_{i}^{\mu} q_{j}^{v} q_{k}^{\lambda} q_{l}^{\sigma} \Pi_{i j k l}^{10}
\end{aligned}
$$

the 95 linearly independent Ward identities

$$
\left\{q_{1 \mu}, q_{2 v}, q_{3 \rho}, q_{4 \sigma}\right\} \Pi^{\mu \nu \lambda \sigma}\left(q_{1}, q_{2}, q_{3}\right)=0
$$

lead to 43 independent gauge-invariant Lorentz tensor structures. The HLbL tensor is also crossing symmetric under the exchange of two momenta, e.g. under

$$
C_{14}=\left\{q_{1} \leftrightarrow-q_{4}, \mu \leftrightarrow \sigma\right\}, \quad C_{13}=\left\{q_{1} \leftrightarrow q_{3}, \mu \leftrightarrow \lambda\right\}
$$

It is usual to introduce a larger set of 54 Lorenz structures, which although redundant, have the properties that the coefficients of the expansion of the HLbL tensor are free of kinematical singularities [34, 35]. This is of the utmost importance, for instance, in the approach based on dispersion relations, which has been recently developed in [36, 37].

Actually, noticing that one of the four photon momenta, say $q_{4}$, corresponding to the external e.m field, vanishes, only $12 \bar{\Pi}_{i}, i=1, \ldots 12$ combinations of the 54 coefficients are needed for the evaluation of the $a_{\mu}^{H l b L}$. Using Gegenbauer polynomials techniques, generalizing those in [11], the authors of Refs.[36, 37] have worked out a Master Formula for $a_{\mu}^{H L b L}$ :

$$
\begin{aligned}
& a_{\mu}^{\mathrm{HLbL}}=\frac{2 \alpha^{3}}{3 \pi^{2}} \int_{0}^{\infty} d Q_{1} \int_{0}^{\infty} d Q_{2} \int_{-1}^{1} d \tau \\
& \sqrt{1-\tau^{2}} Q_{1}^{3} Q_{2}^{3} \sum_{i=1}^{12} \bar{T}_{i}\left(Q_{1}, Q_{2}, \tau\right) \bar{\Pi}_{i}\left(Q_{1}, Q_{2}, \tau\right),
\end{aligned}
$$

where $Q_{1}:=\left|Q_{1}\right|, Q_{2}:=\left|Q_{2}\right|$. The $\bar{\Pi}_{i}$ evaluated for the reduced kinematics

$$
\begin{gathered}
q_{1}^{2}=-Q_{1}^{2}, \quad q_{2}^{2}=-Q_{2}^{2}, \\
q_{3}^{2}=-Q_{3}^{2}=-Q_{1}^{2}-2 Q_{1} Q_{2} \tau-Q_{2}^{2}, \quad q_{4}^{2}=0 .
\end{gathered}
$$

Explicit expressions for the functions $\bar{T}_{i}\left(Q_{1}, Q_{2}, \tau\right)$ can be found in [37].

\subsection{The HLbL tensor in the HS model}

According to the holographic recipe:

$$
\begin{gathered}
\left\langle 0\left|T\left\{J_{e . m .}^{\mu}(x) J_{e . m .}^{v}(y) J_{e . m .}^{\lambda}(z) J_{e . m .}^{\sigma}(0)\right\}\right| 0\right\rangle \\
\sim \frac{\delta^{4} S_{5}}{\delta \mathbf{v}_{e . m .}^{\mu}(x) \delta \mathbf{v}_{e . m .}^{v}(y) \delta \mathbf{v}_{e . m .}^{\lambda}(z) \delta \mathbf{v}_{e . m .}^{\sigma}(0)}
\end{gathered}
$$

vhere $\mathbf{v}_{e . m}^{\mu}(x)$ is the boundary value of the 5D vector gauge field and the 5D action is evaluated on-shell.

For the case of the e.m. currents there is no direct cubic, or quartic interaction terms neither from $S_{Y M}$ nor $S_{C S}$ for obvious reasons. The only possibility amounts to the propagation of the $5 \mathrm{D}$ axial-vector gauge field between two anomalous VVA vertices, generated by the CS term. Taking into account the pion which contributes as zero mode to the axial field, one is led to the two 5D exchange diagrams in Fig.3, together with their crossed version.
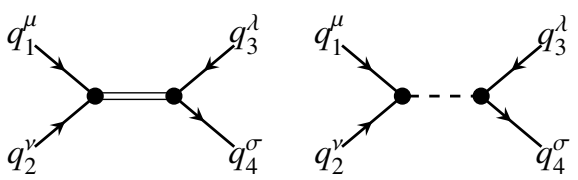

Figure 3. Two of the diagrams contributing to to HLbL tensor in HS model. The diagram on the left contains the 5D axial Green function and accounts for the contribution od the massive axialvector resonances. On the right is the usual one-pion exchange diagram. Two other couples of diagrams are obtained by crossing operations $C_{13}$ and $C_{14}$.

Thus, the key ingredient is the 5D axial Green function

$$
\begin{aligned}
& G_{A}^{\mu v}\left(z, z^{\prime} ; q^{2}\right)= \\
& G_{A}^{T}\left(z, z^{\prime} ; q^{2}\right) P_{T}^{\mu v}(q)+G_{A}^{L}\left(z, z^{\prime}\right) P_{L}^{\mu \nu}(q)
\end{aligned}
$$

where we introduced the transversal and longitudinal tensors

$$
P_{T}^{\mu v}(q)=\left(g^{\mu v}-\frac{q^{\mu} q^{v}}{q^{2}}\right), \quad P_{L}^{\mu v}(q)=\frac{q^{\mu} q^{v}}{q^{2}}
$$

The resulting expression of the HLbL tensor can be written

$$
\Pi^{\mu \nu \lambda \sigma}=\underbrace{\Pi_{L}^{(\pi, A) \mu \nu \lambda \sigma}}_{\text {pion \& massive axial reson. }}+\underbrace{\Pi_{T}^{(A) \mu \nu \lambda \sigma}}_{\text {massive axial reson. }}
$$

where for the massive resonance contribution one has

$$
\begin{aligned}
& \Pi_{L, T}^{(A) \mu \nu \lambda \sigma}=\underbrace{P_{T}^{\mu \mu^{\prime}}\left(q_{1}\right) P_{T}^{v \gamma^{\prime}}\left(q_{2}\right) P_{T}^{\lambda \lambda^{\prime}}\left(q_{3}\right) P_{T}^{\sigma \sigma^{\prime}}\left(q_{4}\right)}_{\text {T proj. on external vector legs }} \\
& \times \underbrace{\varepsilon_{\mu^{\prime} \nu^{\prime} \alpha \beta} \varepsilon_{\lambda^{\prime} \sigma^{\prime} \gamma \delta}}_{\text {anom. couplings }} \times \underbrace{P_{L, T}^{\alpha \gamma} \times \underbrace{A_{L, T}^{\beta \delta}}_{z \text { and } z^{\prime} \text { integrals }}}_{\text {L,T proj. in } G_{A}}
\end{aligned}
$$

$A_{L, T}^{\beta \delta}$ contains combinations of the form $q_{a}^{\beta} q_{c}^{\delta} \mathcal{G}_{A}^{L, T}\left(q_{a}, q_{b} ; q_{c}, q_{d}\right)$ with the convolution integrals

$$
\begin{aligned}
& \mathcal{G}_{A}^{L}\left(q_{a}, q_{b} ; q_{c}, q_{d}\right)= \\
& \begin{aligned}
\int_{0}^{z_{0}} d z \int_{0}^{z_{0}} d z^{\prime} v(z, & \left.q_{a}^{2}\right) \partial_{z} v\left(z, q_{b}^{2}\right) G_{A}^{L}\left(z, z^{\prime}\right) \\
& \times v\left(z^{\prime}, q_{c}^{2}\right) \partial_{z^{\prime}} v\left(z^{\prime}, q_{d}^{2}\right)
\end{aligned}
\end{aligned}
$$

$$
\begin{aligned}
& \mathcal{G}_{A}^{T}\left(q_{a}, q_{b} ; q_{c}, q_{d}\right)= \\
& \int_{0}^{z_{0}} d z \int_{0}^{z_{0}} d z^{\prime} v\left(z, q_{a}^{2}\right) \partial_{z} v\left(z, q_{b}^{2}\right) G_{A}^{T}\left(z, z^{\prime} ; q_{a}+q_{b}\right) \\
& \times v\left(z^{\prime}, q_{c}^{2}\right) \partial_{z^{\prime}} v\left(z^{\prime}, q_{d}^{2}\right)
\end{aligned}
$$


Having constructed a gauge-invariant and cross-symmetric expression for the HLbL tensor, it can be decomposed in the 54 redundant basis and the relevant 12 coefficients $\bar{\Pi}_{i}$ to be used in the Master Equation for $a_{\mu}$. In this way we can extract the value of $a_{\mu}$ predicted by the HS model.

More importantly than the numerical value of $a_{\mu}$, is, however, the study of the asymptotic behaviour of the HLbL tensor obtained.

As discussed in the Introduction, HQCD models are taylored to match the leading behaviour of pQCD in the deep Euclidean region of momenta. This was the case for the two point function, reproducing the parton loop log, and for the three point function in the case of the anomalous pion TFF, where the correct powers in $1 / Q^{2}$ were obtained for single and double photon large Euclidean virtualities.

The same question can be raised for the four-point function represented by the HLbL tensor.

Focusing on the longitudinal part of the HLbL tensor (similar constraints can be written for the transversal part), the MV constraint is a particular case of a more general non renormalization theorem [38, 39], which holds in a particular kinematic limit

$$
\begin{aligned}
& \Pi_{\mu \nu \lambda \sigma}^{L}\left(\lambda q_{1}, q_{2}-\lambda q_{1}, q_{3}\right) \underset{\lambda \rightarrow \infty}{\sim} \frac{1}{\lambda} \frac{\alpha}{9 \pi^{2}} \varepsilon_{\mu \nu \alpha \beta} \varepsilon_{\lambda \sigma \gamma \delta} \\
& \times \frac{q_{1}^{\alpha} q_{2}^{\beta} q_{2}^{\gamma} q_{3}^{\delta}}{q_{2}^{2}} \sum_{a=0,3,8} C_{a} w^{L(a)}\left(\left(q_{1}+q_{3}\right)^{2}, q_{3}^{2}, q_{2}^{2}\right)
\end{aligned}
$$

where $w^{L(a)}$ is the coefficient longitudinal tensor structure part of the three-point AVV function (with the $C_{a}$ some isospin factors):

$$
\mathcal{W}_{\mu \nu \rho}^{(a)}\left(q_{1}, q_{2}\right) \equiv i \int d^{4} x d^{4} y e^{i\left(q_{1} x+q_{2} y\right)}\left\langle\left|T\left\{j_{\mu}(x) j_{v}(y)\right\}\right|\right\rangle .
$$

In the chiral limit $m_{\pi}=0$, Adler-Bell-Jackiw anomaly completely determine, i.e. without perturbative and non perturbative corrections, the value of $w^{L}$ :

$$
w^{L(a)}\left(\left(q_{1}+q_{3}\right)^{2}, q_{3}^{2}, q_{2}^{2}\right)=-\frac{2 N_{c}}{q_{3}^{2}}
$$

In the case of the soft photon, as for HLbL diagrams, the constraint extends to the transverse part of the AVV function

$$
w^{L(a)}\left(0, q_{3}^{2}, 0\right)=2 w^{L(a)}\left(0, q_{3}^{2}, 0\right)=-\frac{2 N_{c}}{q_{3}^{2}}
$$

It can be shown that the expression of the HLbL obtained in the HS model satisfies thye MV constraint (9), due to a peculiar interplay between the contributions of the pion and of axial vector exchange. While the pion contribution is dominating at low momenta, the massive axial resonance contribution gives the MV behaviour for Large Euclidean momenta, and is the sole responsible for the saturation of the anomaly in (9) in that kinematical region.

This is an important result, because in phenomenological models based only one the one-pion exchange, it was impossible to satisfy the MV constraint, unless making very ad hoc hypothesis. For instance, MV proposed to fullfill the constraint using pointlike WZW at the vertex with physical external photon, while JN got the MV behaviour using LMD+V TFF's, with a elaborate choice of the parameters, beyond the minimal set required by chiral symmetry or Large-Nc. Most importantly, all attempts to comply with the MV constraint, lead to an increase of the accepted estimate of the HLbL conthribution to $a_{\mu}$, and this has significant impact on phenomenology.

The HS model that we have studied seems to be the first field theoretical model to satisfy MV constraint, without any extra assumption, and that's why, despite its simplicity, it is worth to be studied. It affirms that there is a a definite prediction for the contribution of the (full tower of) massive axial vector resonances to the one-particle exchange diagram of HLbL, and allows a preliminary numerical analysis, which depend on the choice of the parameters of the model. For the choice that we madeset of parameter used in the evaluation of the one-pion exchange contribution, in Sec. 4, one gets for the total contributions, obtained summing over the three different isospin channels of pseudoscalar $\left(\pi_{0}, \eta\right.$ and $\left.\eta^{\prime}\right)$ and axial lvector resonances, the values:

$$
a_{\mu}^{P S}=8.2 \times 10^{-10}, \quad a_{\mu}^{P V}=2.8 \times 10^{-10}
$$

Other choices of the parameters of the model produce similar results, with the one-pion exchange always being responsible for the bulk of the numerical result. The total contribution of the axial vector resonances contribution turns out to be bigger than those claimed in the literature $[40,41]$.

\section{Conclusions}

Holographic models of QCD, are a valuable laboratory in which, using analytic and semi-analytic method, non perturbative properties of real QCD can be simulated and tested in a simpler way. Although these models can be refined, trying to improve their predictive power, their main value lies in the the opportunity they offer to discover hints of non perturbative properties of real $\mathrm{QCD}$, to be tested with more powerful approaches.

We have illustrated the main ingredients of one particular model, introduced by Hirn and Sanz, and shown how one can evaluate the one-pion exchange contribution, by mean of the expression of the pion transition form factor provided by the model.

Then, we turned to a direct study of the Hadronic Light-by-Light tensor, presenting some preliminary result which will be more systematically described in a forthcoming paper. We focused on the contribution of the infinite tower of massive axial-vector resonances, which are encoded in the 5D axial-vector Green function, and contains both a longitudinal and a transverse part. The longitudinal part produces the same tensor structures of the HLbL tensor which are generated by the one-pion exchange diagrams. When added together, the two contributions, the one due to the one-pion exchange and the one due to the longitudinal part of the 5D axial-vector Green 
function, play a dual role, dominating the one on the other at low and at high momenta respectively.

In particular, it is the contributions of the longitudinal axial-vector Green functions, which dominates for deep Euclidean momenta and allows the resulting amplitude to satisfy the UV costraint derived by Melnikov and Vainshtein using QCD OPE.

The transversal part of the 5D axial-vector Green function, gives non vanishing coefficients for a subset of tensor structures, complementary to those of the longitudinal part. Both the longitudinal and the transversal contribution due to the axial-vector vanishes at zero momenta, but have the same mild decreasing behaviour in the deep Euclidean region to satisfy the MV constraint.

We stress the fact that MV constraints are naturally fulfilled in the HQCD model, which contains only few parameters, without any further assumption.

Preliminary numerical analysis of the of values obtained for $a_{\mu}$ has also been shown.

Note Added in Proof. After the presentation of this talk and before [26] was completed, interesting papers on the effect of QCD short distance constraints on the HLbL contribution appeared. In Refs. $[42,43]$ the effects on the longitudinal part were discussed in the framework of the dispersion relations and attributed to an infinite tower of massive pseudoscalars. Such approach was criticized in [44]. The authors of [45] independently discovered that the MV constraints can be fulfilled using holographic models with infinite tower of massive axial vectors.

\section{Aknowledgments}

I thank the organizers of the Anacapri FCCP 2019 Workshop for the invitation and for the stimulating atmosphere. I thank O. Cata, G.D'Ambrosio, D.Greynat and A. Iyer for the long-standing scientific collaboration, which deeply sharpened my understanding of the subject. I was supported in part by MIUR under Project No. 2015P5SBHT and by the INFN research initiative ENP.

\section{References}

[1] M. Tanabashi et al. (Particle Data Group), Phys. Rev. D 98, 030001 (2018)

[2] G. Venanzoni [Fermilab E989 Collaboration], Nucl. Part. Phys. Proc. 273-275, 584 (2016) [arXiv:1411.2555].

[3] M. Otani [E34 Collaboration], JPS Conf. Proc. 8, 025010 (2015).

[4] F. Jegerlehner and A. Nyffeler, Phys. Rept. 477, 1 (2009) [arXiv:0902.3360 [hep-ph]].

[5] J. Bijnens, E. Pallante and J. Prades, Phys. Rev. Lett. 75, 1447 (1995) [Phys. Rev. Lett. 75, 3781 (1995)] [hep-ph/9505251].

[6] J. Bijnens, E. Pallante and J. Prades, Nucl. Phys. B 474, 379 (1996) [hep-ph/9511388].

[7] M. Hayakawa, T. Kinoshita and A. I. Sanda, Phys. Rev. Lett. 75, 790 (1995) [hep-ph/9503463].
[8] M. Hayakawa, T. Kinoshita and A. I. Sanda, Phys. Rev. D 54, 3137 (1996) [hep-ph/9601310].

[9] M. Hayakawa and T. Kinoshita, Phys. Rev. D 57, 465 (1998) [Phys. Rev. D 66, 019902 (2002)] [hep$\mathrm{ph} / 9708227]$.

[10] M. Hayakawa and T. Kinoshita, [ hep-ph/0112102].

[11] M. Knecht and A. Nyffeler, Phys. Rev. D 65, 073034 (2002) [arXiv:hep-ph/0111058].

[12] M. Knecht, A. Nyffeler, M. Perrottet and E. de Rafael, Phys. Rev. Lett. 88, 071802 (2002) [hepph/0111059].

[13] M. Knecht, S. Peris, M. Perrottet and E. de Rafael, Phys. Rev. Lett. 83, 5230 (1999) [hep-ph/9908283].

[14] M. Knecht and A. Nyffeler, Eur. Phys. J. C 21, 659 (2001) [hep-ph/0106034].

[15] K. Melnikov and A. Vainshtein, Phys. Rev. D 70, 113006 (2004) [hep-ph/0312226].

[16] J. Prades, E. de Rafael and A. Vainshtein, Adv. Ser. Direct. High Energy Phys. 20, 303 (2009) [arXiv:0901.0306 [hep-ph]].

[17] T. Sakai and S. Sugimoto, Prog. Theor. Phys. 113, 843 (2005). [hep-th/0412141].

[18] J. Erlich, E. Katz, D. T. Son and M. A. Stephanov, Phys. Rev. Lett. 95, 261602 (2005). [arXiv:hep$\mathrm{ph} / 0501128]$.

[19] L. Da Rold and A. Pomarol, Nucl.Phys. B721 (2005) 79; [hep-ph/0501218].

[20] J. Hirn and V. Sanz, JHEP 0512, 030 (2005); [hep$\mathrm{ph} / 0507049]$.

[21] A. Karch, E. Katz, D. T. Son and M. A. Stephanov, [arXiv:hep-ph/0602229].

[22] D. K. Hong, D. Kim and S. Matsuzaki, Phys. Rev. D 81, 073005 (2010) [arXiv:0911.0560 [hep-ph]].

[23] L. Cappiello, O. Cata and G. D’Ambrosio, Phys. Rev. D 83, 093006 (2011) [arXiv:1009.1161 [hep$\mathrm{ph}]]$.

[24] T. Hambye, B. Hassanain, J. March-Russell and M. Schvellinger, Phys. Rev. D 74, 026003 (2006) [hep-ph/0512089].

[25] T. Hambye, B. Hassanain, J. March-Russell and M. Schvellinger, Phys. Rev. D 76, 125017 (2007) [hep-ph/0612010].

[26] L. Cappiello, O. Catà, G. D. Ambrosio, D. Greynat and A. Iyer, arXiv:1912.02779 [hep-ph].

[27] J. M. Maldacena, Adv. Theor. Math. Phys. 2, 231 (1998).

[28] S. S. Gubser, I. R. Klebanov and A. M. Polyakov, Phys. Lett. B 428, 105 (1998).

[29] E. Witten, Adv. Theor. Math. Phys. 2, 253 (1998).

[30] M. Tanabashi et al. [Particle Data Group], "Review of Particle Physics,” Phys. Rev. D 98, no. 3, 030001 (2018).

[31] J. Leutgeb, J. Mager and A. Rebhan, Phys. Rev. D 100, no. 9, 094038 (2019) [arXiv:1906.11795 [hep$\mathrm{ph}]]$.

[32] I. Danilkin, C. F. Redmer and M. Vanderhaeghen, Prog. Part. Nucl. Phys. 107, 20 (2019) 
[arXiv:1901.10346 [hep-ph]].

[33] A. Gérardin, H. B. Meyer and A. Nyffeler, Phys. Rev. D 100, no. 3, 034520 (2019) [arXiv:1903.09471 [hep-lat]].

[34] W. A. Bardeen and W. K. Tung, Phys. Rev. 173, 1423 (1968), [Erratum: Phys. Rev. D4, 3229 (1971)].

[35] R. Tarrach, Nuovo Cim. A28, 409 (1975).

[36] G. Colangelo, M. Hoferichter, M. Procura and P. Stoffer, JHEP 1509, 074 (2015) [arXiv:1506.01386 [hep-ph]].

[37] G. Colangelo, M. Hoferichter, M. Procura and P. Stoffer, JHEP 1704, 161 (2017) [arXiv:1702.07347 [hep-ph]].

[38] A. Vainshtein, Phys. Lett. B 569, 187 (2003) [hep$\mathrm{ph} / 0212231]$.
[39] M. Knecht, S. Peris, M. Perrottet and E. de Rafael, JHEP 0403, 035 (2004) [hep-ph/0311100].

[40] F. Jegerlehner, EPJ Web Conf. 199, 01010 (2019) [arXiv:1809.07413 [hep-ph]].

[41] V. Pauk and M. Vanderhaeghen, Eur. Phys. J. C 74, no. 8, 3008 (2014) [arXiv:1401.0832 [hep-ph]].

[42] G. Colangelo, F. Hagelstein, M. Hoferichter, L. Laub and P. Stoffer, arXiv:1910.13432 [hep-ph].

[43] G. Colangelo, F. Hagelstein, M. Hoferichter, L. Laub and P. Stoffer, arXiv:1910.11881 [hep-ph].

[44] K. Melnikov and A. Vainshtein, arXiv:1911.05874 [hep-ph].

[45] J. Leutgeb and A. Rebhan, arXiv:1912.01596 [hep$\mathrm{ph}]$. 